\title{
Search for scalar top and scalar bottom quarks at LEP
}

\section{OPAL Collaboration}

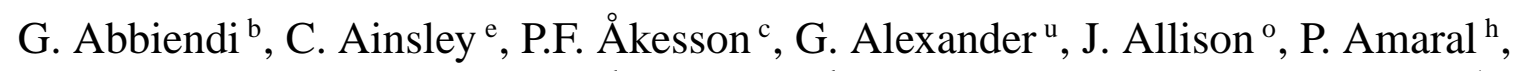
G. Anagnostou a , K.J. Anderson ${ }^{\text {h}}$, S. Arcelli ${ }^{\text {b }}$, S. Asai ${ }^{\text {}}$, D. Axen ${ }^{\mathrm{z}}$, G. Azuelos ${ }^{\mathrm{q}, 1}$, I. Bailey ${ }^{\mathrm{y}}$, E. Barberio ${ }^{\mathrm{g}}$, R.J. Barlow ${ }^{\mathrm{o}}$, R.J. Batley ${ }^{\mathrm{e}}$, P. Bechtle ${ }^{\mathrm{x}}$, T. Behnke ${ }^{\mathrm{x}}$, K.W. Bell ${ }^{\text {s }}$, P.J. Bell ${ }^{\text {a }}$, G. Bella ${ }^{\text {u }}$, A. Bellerive ${ }^{\mathrm{f}}$, G. Benelli $^{\mathrm{d}}$, S. Bethke ${ }^{\mathrm{ae}}$, O. Biebel $^{\text {ae }}$, I.J. Bloodworth ${ }^{\text {a }}$, O. Boeriu ${ }^{\mathrm{i}}$, P. Bock ${ }^{\mathrm{j}}$, D. Bonacorsi ${ }^{\mathrm{b}}$, M. Boutemeur ${ }^{\text {ad }}$, S. Braibant ${ }^{\mathrm{g}}$, L. Brigliadori ${ }^{\text {b }}$, R.M. Brown ${ }^{\mathrm{s}}$, K. Buesser ${ }^{\mathrm{x}}$, H.J. Burckhart ${ }^{\mathrm{g}}$, S. Campana ${ }^{\mathrm{d}}$, R.K. Carnegie ${ }^{\text {f }}$, B. Caron ${ }^{\text {aa }}$, A.A. Carter ${ }^{1}$, J.R. Carter ${ }^{\mathrm{e}}$, C.Y. Chang ${ }^{\mathrm{p}}$, D.G. Charlton ${ }^{\mathrm{a}, 2}$, A. Csilling g, ${ }^{\mathrm{g}}$, M. Cuffiani ${ }^{\mathrm{b}}$, S. Dado ${ }^{\mathrm{t}}$, G.M. Dallavalle ${ }^{\mathrm{b}}$, S. Dallison ${ }^{\mathrm{o}}$, A. De Roeck ${ }^{\mathrm{g}}$, E.A. De Wolf ${ }^{g}$, K. Desch ${ }^{\text {x }}$, B. Dienes ${ }^{\text {ac }}$, M. Donkers ${ }^{\text {f }}$, J. Dubbert ${ }^{\text {ad }}$, E. Duchovni ${ }^{\text {w }}$, G. Duckeck ${ }^{\text {ad }}$, I.P. Duerdoth ${ }^{\text {o }}$, E. Elfgren ${ }^{\mathrm{q}}$, E. Etzion ${ }^{\mathrm{u}}$, F. Fabbri ${ }^{\mathrm{b}}$, L. Feld ${ }^{\mathrm{i}}$, P. Ferrari ${ }^{\mathrm{g}}$, F. Fiedler ${ }^{\text {ad }}$, I. Fleck ${ }^{\mathrm{i}}$, M. Ford ${ }^{\mathrm{e}}$, A. Frey ${ }^{\mathrm{g}}$, A. Fürtjes ${ }^{\mathrm{g}}$, P. Gagnon ${ }^{\mathrm{k}}$, J.W. Gary ${ }^{\mathrm{d}}$, G. Gaycken ${ }^{\text {x }}$, C. Geich-Gimbel ${ }^{\text {c }}$, G. Giacomelli ${ }^{\text {b }}$, P. Giacomelli ${ }^{\text {b }}$, M. Giunta ${ }^{\text {d, }}$ J. Goldberg ${ }^{\text {, }}$, E. Gross ${ }^{\text {w }}$, J. Grunhaus ${ }^{\text {u, M. Gruwé }}{ }^{\mathrm{g}}$, P.O. Günther ${ }^{\mathrm{c}}$, A. Gupta ${ }^{\text {, }}$, C. Hajdu ${ }^{\text {ab }}$, M. Hamann ${ }^{\text {x }}$, G.G. Hanson ${ }^{\text {d }}$, K. Harder ${ }^{\text {, }}$, A. Harel ${ }^{\text {t }}$, M. Harin-Dirac ${ }^{\text {d, }}$, M. Hauschild ${ }^{\mathrm{g}}$, J. Hauschildt ${ }^{\mathrm{x}}$, C.M. Hawkes ${ }^{\mathrm{a}}$, R. Hawkings ${ }^{\mathrm{g}}$, R.J. Hemingway ${ }^{\mathrm{f}}$, C. Hensel ${ }^{\mathrm{x}}$, G. Herten ${ }^{\mathrm{i}}$, R.D. Heuer ${ }^{\mathrm{x}}$, J.C. Hill ${ }^{\mathrm{e}}$, K. Hoffman ${ }^{\mathrm{h}}$, R.J. Homer ${ }^{\mathrm{a}}$, D. Horváth ${ }^{\mathrm{ab}, 3}$, R. Howard ${ }^{\mathrm{z}}$, P. Hüntemeyer ${ }^{\mathrm{x}}$, P. Igo-Kemenes ${ }^{\mathrm{j}}$, K. Ishii ${ }^{\mathrm{v}}$, H. Jeremie ${ }^{\mathrm{q}}$, P. Jovanovic ${ }^{\text {a }}$, T.R. Junk ${ }^{\text {f }}$, N. Kanaya ${ }^{y}$, J. Kanzaki ${ }^{\text {}}$, G. Karapetian ${ }^{\text {q }}$, D. Karlen ${ }^{f}$, V. Kartvelishvili ${ }^{\text {o }}$, K. Kawagoe ${ }^{\text {v }}$, T. Kawamoto ${ }^{\text {}}$, R.K. Keeler ${ }^{\mathrm{y}}$, R.G. Kellogg ${ }^{\text {p }}$, B.W. Kennedy ${ }^{\text {s, D.H. Kim }}{ }^{\mathrm{r}}$, K. Klein ${ }^{\mathrm{j}}$, A. Klier ${ }^{\mathrm{w}}$, S. Kluth ${ }^{\mathrm{ae}}$, T. Kobayashi ${ }^{\mathrm{v}}$, M. Kobel ${ }^{\text {c }, ~ S . ~ K o m a m i y a ~}{ }^{v}$, L. Kormos ${ }^{y}$, R.V. Kowalewski ${ }^{y}$, T. Krämer ${ }^{\text {x }}$, T. Kress ${ }^{\text {d, }}$ P. Krieger ${ }^{\text {f,12 }}$, J. von Krogh ${ }^{\mathrm{j}}$, D. Krop ${ }^{\mathrm{k}}$, K. $\operatorname{Kruger}^{\mathrm{g}}$, M. Kupper ${ }^{\mathrm{w}}$, G.D. Lafferty ${ }^{\mathrm{o}}$, H. Landsman ${ }^{\mathrm{t}}$, D. Lanske ${ }^{\mathrm{m}}$, J.G. Layter ${ }^{\mathrm{d}}$, A. Leins ${ }^{\text {ad }}$, D. Lellouch ${ }^{\mathrm{w}}$, J. Letts ${ }^{\mathrm{k}}$,

L. Levinson ${ }^{\text {w }}$, J. Lillich ${ }^{\mathrm{i}}$, S.L. Lloyd ${ }^{1}$, F.K. Loebinger ${ }^{\mathrm{o}}$, J. Lu ${ }^{\mathrm{z}}$, J. Ludwig ${ }^{\mathrm{i}}$,

A. Macpherson ${ }^{\text {aa, } 9}$, W. Mader ${ }^{\text {c }}$, S. Marcellini ${ }^{\text {b }}$, T.E. Marchant ${ }^{\circ}$, A.J. Martin ${ }^{1}$, J.P. Martin ${ }^{\mathrm{q}}$, G. Masetti ${ }^{\text {b }}$, T. Mashimo ${ }^{\text {v }}$, P. Mättig ${ }^{13}$, W.J. McDonald ${ }^{\text {aa }}$, J. McKenna ${ }^{\mathrm{z}}$, T.J. McMahon ${ }^{\mathrm{a}}$, R.A. McPherson ${ }^{\mathrm{y}}$, F. Meijers ${ }^{\mathrm{g}}$, P. Mendez-Lorenzo ${ }^{\text {ad }}$, W. Menges ${ }^{\mathrm{x}}$, F.S. Merritt ${ }^{\text {h }}$, H. Mes ${ }^{\text {f, }}$, A. Michelini ${ }^{\text {b }}$, S. Mihara ${ }^{\text {v }}$, G. Mikenberg ${ }^{\text {w }}$, D.J. Miller ${ }^{\mathrm{n}}$, S. Moed ${ }^{\text {t }}$ W. Mohr ${ }^{\text {i }}$, T. Mori ${ }^{\mathrm{v}}$, A. Mutter ${ }^{\mathrm{i}}$, K. Nagai ${ }^{1}$, I. Nakamura ${ }^{\mathrm{v}}$, H.A. Neal ${ }^{\text {af }}$, 
R. Nisius ${ }^{\mathrm{g}}$, S.W. O’Neale ${ }^{\mathrm{a}}$, A. Oh ${ }^{\mathrm{g}}$, A. Okpara ${ }^{\mathrm{j}}$, M.J. Oreglia ${ }^{\mathrm{h}}$, S. Orito ${ }^{\mathrm{v}}$, C. Pahl ${ }^{\text {ae }}$, G. Pásztor ${ }^{\mathrm{d}, 7}$, J.R. Pater ${ }^{\mathrm{o}}$, G.N. Patrick ${ }^{\mathrm{s}}$, J.E. Pilcher ${ }^{\mathrm{h}}$, J. Pinfold ${ }^{\text {aa }}$, D.E. Plane ${ }^{\mathrm{g}}$,

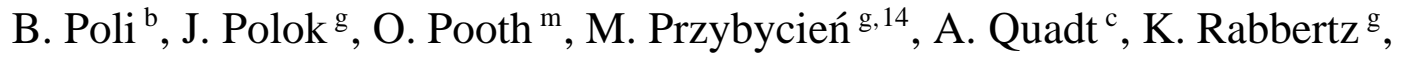
C. Rembser ${ }^{\mathrm{g}}$, P. Renkel ${ }^{\mathrm{w}}$, H. Rick ${ }^{\mathrm{d}}$, J.M. Roney ${ }^{\mathrm{y}}$, S. Rosati ${ }^{\mathrm{c}}$, Y. Rozen ${ }^{\mathrm{t}}$, K. Runge ${ }^{\mathrm{i}}$, K. Sachs ${ }^{\text {f }}$, T. Saeki ${ }^{\text {v }}$, O. Sahr ${ }^{\text {ad }}$, E.K.G. Sarkisyan ${ }^{\text {g, } 10}$, A.D. Schaile ${ }^{\text {ad }}$, O. Schaile ${ }^{\text {ad }}$, P. Scharff-Hansen ${ }^{\mathrm{g}}$, J. Schieck ${ }^{\text {ae }}$, T. Schoerner-Sadenius ${ }^{\mathrm{g}}$, M. Schröder ${ }^{\mathrm{g}}$,

M. Schumacher ${ }^{\text {c }}$, C. Schwick ${ }^{g}$, W.G. Scott ${ }^{\mathrm{s}}$, R. Seuster ${ }^{\mathrm{m}, 6}$, T.G. Shears ${ }^{\mathrm{g}, 8}$, B.C. Shen ${ }^{\mathrm{d}}$, C.H. Shepherd-Themistocleous ${ }^{\mathrm{e}}$, P. Sherwood ${ }^{\mathrm{n}}$, G. Siroli ${ }^{\mathrm{b}}$, A. Skuja ${ }^{\mathrm{p}}$, A.M. Smith ${ }^{\mathrm{g}}$, R. Sobie ${ }^{y}$, S. Söldner-Rembold ${ }^{\mathrm{i}, 4}$, S. Spagnolo ${ }^{\mathrm{s}}$, F. Spano ${ }^{\text {h}}$, A. Stahl ${ }^{\mathrm{c}}$, K. Stephens ${ }^{\circ}$, D. Strom ${ }^{\mathrm{r}}$, R. Ströhmer ${ }^{\text {ad }}$, S. Tarem ${ }^{\mathrm{t}}$, M. Tasevsky ${ }^{\mathrm{g}}$, R.J. Taylor ${ }^{\mathrm{n}}$, R. Teuscher ${ }^{\mathrm{h}}$, M.A. Thomson ${ }^{\mathrm{e}}$, E. Torrence ${ }^{\mathrm{r}}$, D. Toya ${ }^{\mathrm{v}}$, P. Tran ${ }^{\mathrm{d}}$, T. Trefzger $^{\text {ad }}$, A. Tricoli ${ }^{\mathrm{b}}$, I. Trigger ${ }^{\mathrm{g}}$, Z. Trócsányi ${ }^{\text {ac, } 5}$, E. Tsur ${ }^{\mathrm{u}}$, M.F. Turner-Watson ${ }^{\mathrm{a}}$, I. Ueda ${ }^{\mathrm{v}}$, B. Ujvári ${ }^{\text {ac }, 5}$, B. Vachon ${ }^{\mathrm{y}}$, C.F. Vollmer ${ }^{\text {ad }}$, P. Vannerem ${ }^{\mathrm{i}}$, M. Verzocchi ${ }^{\mathrm{p}}$, H. Voss ${ }^{\mathrm{g}}$, J. Vossebeld ${ }^{\mathrm{g}, 8}$, D. Waller ${ }^{\text {f }}$, C.P. Ward ${ }^{\text {e }}$, D.R. Ward ${ }^{\text {e }}$, P.M. Watkins ${ }^{\text {a }}$, A.T. Watson ${ }^{\text {a }}$, N.K. Watson ${ }^{\text {a }}$, P.S. Wells ${ }^{\mathrm{g}}$, T. Wengler ${ }^{\mathrm{g}}$, N. Wermes ${ }^{\mathrm{c}}$, D. Wetterling ${ }^{\mathrm{j}}$, G.W. Wilson ${ }^{\mathrm{o}, 11}$, J.A. Wilson ${ }^{\mathrm{a}}$, G. Wolf ${ }^{\mathrm{w}}$, T.R. Wyatt ${ }^{\mathrm{o}}$, S. Yamashita ${ }^{\mathrm{v}}$, D. Zer-Zion ${ }^{\mathrm{d}}$, L. Zivkovic ${ }^{\mathrm{w}}$

\footnotetext{
${ }^{a}$ School of Physics and Astronomy, University of Birmingham, Birmingham B15 2TT, UK

b Dipartimento di Fisica dell' Università di Bologna and INFN, I-40126 Bologna, Italy ${ }^{\mathrm{c}}$ Physikalisches Institut, Universität Bonn, D-53115 Bonn, Germany

${ }^{\mathrm{d}}$ Department of Physics, University of California, Riverside, CA 92521, USA e Cavendish Laboratory, Cambridge CB3 OHE, UK

f Ottawa-Carleton Institute for Physics, Department of Physics, Carleton University, Ottawa, Ontario K1S 5B6, Canada g CERN, European Organisation for Nuclear Research, CH-1211 Geneva 23, Switzerland

${ }^{\mathrm{h}}$ Enrico Fermi Institute and Department of Physics, University of Chicago, Chicago, IL 60637, USA

${ }^{\mathrm{i}}$ Fakultät für Physik, Albert-Ludwigs-Universität Freiburg, D-79104 Freiburg, Germany

j Physikalisches Institut, Universität Heidelberg, D-69120 Heidelberg, Germany

${ }^{\mathrm{k}}$ Indiana University, Department of Physics, Swain Hall West 117, Bloomington, IN 47405, USA

${ }^{1}$ Queen Mary and Westfield College, University of London, London E1 4NS, UK

${ }^{\mathrm{m}}$ Technische Hochschule Aachen, III Physikalisches Institut, Sommerfeldstrasse 26-28, D-52056 Aachen, Germany ${ }^{\mathrm{n}}$ University College London, London WC1E 6BT, UK

${ }^{\circ}$ Department of Physics, Schuster Laboratory, The University, Manchester M13 9PL, UK

p Department of Physics, University of Maryland, College Park, MD 20742, USA

q Laboratoire de Physique Nucléaire, Université de Montréal, Montréal, Quebec H3C 3J7, Canada

${ }^{\mathrm{r}}$ University of Oregon, Department of Physics, Eugene, OR 97403, USA

${ }^{\mathrm{s}}$ CLRC Rutherford Appleton Laboratory, Chilton, Didcot, Oxfordshire OX11 0QX, UK

${ }^{\mathrm{t}}$ Department of Physics, Technion-Israel Institute of Technology, Haifa 32000, Israel

u Department of Physics and Astronomy, Tel Aviv University, Tel Aviv 69978, Israel

${ }^{v}$ International Centre for Elementary Particle Physics and Department of Physics, University of Tokyo, Tokyo 113-0033, and Kobe University, Kobe 657-8501, Japan

${ }^{w}$ Particle Physics Department, Weizmann Institute of Science, Rehovot 76100, Israel

${ }^{\mathrm{x}}$ Universität Hamburg/DESY, Institut für Experimentalphysik, Notkestrasse 85, D-22607 Hamburg, Germany

${ }^{\mathrm{y}}$ University of Victoria, Department of Physics, PO Box 3055, Victoria BC V8W 3P6, Canada

${ }^{\mathrm{z}}$ University of British Columbia, Department of Physics, Vancouver BC V6T 1Z1, Canada

${ }^{\text {aa }}$ University of Alberta, Department of Physics, Edmonton AB T6G 2J1, Canada

${ }^{\text {ab }}$ Research Institute for Particle and Nuclear Physics, PO Box 49, H-1525 Budapest, Hungary

${ }^{a c}$ Institute of Nuclear Research, PO Box 51, H-4001 Debrecen, Hungary

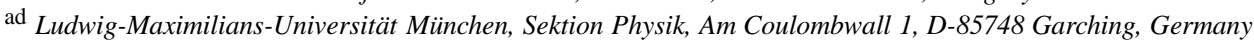

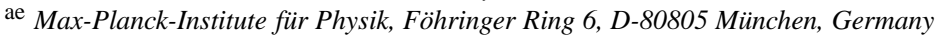


af Yale University, Department of Physics, New Haven, CT 06520, USA

Received 12 July 2002; received in revised form 26 August 2002; accepted 28 August 2002

Editor: L. Montanet

\begin{abstract}
Searches for a scalar top quark and a scalar bottom quark have been performed using a data sample of $438 \mathrm{pb}^{-1}$ at centreof-mass energies of $\sqrt{s}=192-209 \mathrm{GeV}$ collected with the OPAL detector at LEP. No evidence for a signal was found. The $95 \%$ confidence level lower limit on the scalar top quark mass is $97.6 \mathrm{GeV}$ if the mixing angle between the supersymmetric partners of the left- and right-handed states of the top quark is zero. When the scalar top quark decouples from the $\mathrm{Z}^{0}$ boson, the lower limit is $95.7 \mathrm{GeV}$. These limits were obtained assuming that the scalar top quark decays into a charm quark and the lightest neutralino, and that the mass difference between the scalar top quark and the lightest neutralino is larger than $10 \mathrm{GeV}$. The complementary decay mode of the scalar top quark decaying into a bottom quark, a charged lepton and a scalar neutrino has also been studied. The lower limit on the scalar top quark mass is $96.0 \mathrm{GeV}$ for this decay mode, if the mass difference between the scalar top quark and the scalar neutrino is greater than $10 \mathrm{GeV}$ and if the mixing angle of the scalar top quark is zero. From a search for the scalar bottom quark, a mass limit of $96.9 \mathrm{GeV}$ was obtained if the mass difference between the scalar bottom quark and the lightest neutralino is larger than $10 \mathrm{GeV}$.
\end{abstract}

() 2002 Elsevier Science B.V. All rights reserved.

\section{Introduction}

Supersymmetric (SUSY) extensions of the Standard Model predict the existence of bosonic partners of all known fermions. The scalar top quark $(\tilde{\mathfrak{t}})$, which is the bosonic partner of the top quark, may be light because of supersymmetric radiative corrections [1].

E-mail address: david.plane@cern.ch (D.E. Plane).

1 And at TRIUMF, Vancouver, Canada V6T 2A3.

2 And Royal Society University Research Fellow.

3 And Institute of Nuclear Research, Debrecen, Hungary.

4 And Heisenberg Fellow.

5 And Department of Experimental Physics, Lajos Kossuth University, Debrecen, Hungary.

6 And MPI München.

7 And Research Institute for Particle and Nuclear Physics, Budapest, Hungary.

8 Now at University of Liverpool, Department of Physics, Liverpool L69 3BX, UK.

9 And CERN, EP Div, 1211 Geneva 23.

10 And Universitaire Instelling Antwerpen, Physics Department, B-2610 Antwerpen, Belgium.

11 Now at University of Kansas, Department of Physics and Astronomy, Lawrence, KS 66045, USA.

12 Now at University of Toronto, Department of Physics, Toronto, Canada.

13 Current address: Bergische Universität, Wuppertal, Germany.

14 And University of Mining and Metallurgy, Cracow, Poland.
Furthermore, the supersymmetric partners of the righthanded and left-handed top quarks ( $\tilde{t}_{R}$ and $\tilde{t}_{L}$ ) mix, and the resulting two mass eigenstates $\left(\tilde{\mathrm{t}}_{1}\right.$ and $\left.\tilde{\mathrm{t}}_{2}\right)$ have a mass splitting which may be very large due to the large top quark mass. The resulting lighter mass eigenstate $\left(\tilde{\mathfrak{t}}_{1}\right), \tilde{\mathfrak{t}}_{1}=\tilde{\mathfrak{t}}_{\mathrm{L}} \cos \theta_{\tilde{\mathfrak{t}}}+\tilde{\mathfrak{t}}_{\mathrm{R}} \sin \theta_{\tilde{\mathrm{t}}}$, where $\theta_{\tilde{\mathrm{t}}}$ is a mixing angle, can be lighter than any other charged SUSY particle, and also lighter than the top quark [1]. All SUSY breaking parameters are absorbed in $\theta_{\tilde{\mathfrak{t}}}$ and the mass of $\tilde{\mathrm{t}}_{1}$.

The scalar bottom quark ( $\tilde{b})$ can also be light if $\tan \beta$, the ratio of vacuum expectation values of the two Higgs doublet fields, is large. In this case, the analogous mixing between the supersymmetric partners of the right- and left-handed states of the bottom quark $\left(\tilde{b}_{\mathrm{R}}\right.$ and $\left.\tilde{b}_{\mathrm{L}}\right)$ becomes large, and the resulting two mass eigenstates $\left(\tilde{b}_{1}\right.$ and $\left.\tilde{b}_{2}\right)$ also have a large mass splitting [2]. The mass of the lighter mass eigenstate $\left(\tilde{b}_{1}\right)$ may, therefore, be within the reach of LEP.

Assuming R-parity [3] conservation and that the $\tilde{\chi}_{2}^{0}$ and $\tilde{\ell}^{ \pm}$are heavier than the $\tilde{\mathfrak{t}}_{1}$, the dominant decay mode of the $\tilde{\mathrm{t}}_{1}$ is expected to be either $\tilde{\mathrm{t}}_{1} \rightarrow \mathrm{c} \tilde{\chi}_{1}^{0}$ or $\tilde{\mathrm{t}}_{1} \rightarrow \mathrm{b} \tilde{\mathrm{v}} \ell^{+}$, where $\tilde{\chi}_{1}^{0}$ is the lightest neutralino, $\tilde{v}$ is the scalar neutrino, and $\ell$ is e, $\mu$ or $\tau$. The latter decay mode is dominant if it is kinematically allowed. Otherwise the flavour changing two-body 
decay, $\tilde{\mathrm{t}}_{1} \rightarrow \mathrm{c} \tilde{\chi}_{1}^{0}$, is dominant except for the small region where $m_{\tilde{\mathrm{t}}_{1}}-m_{\tilde{\chi}_{1}^{0}}>m_{\mathrm{W}^{ \pm}}+m_{\mathrm{b}} .{ }^{15}$ Both of these decay modes $\left(\tilde{\mathrm{t}}_{1} \rightarrow \mathrm{c} \tilde{\chi}_{1}^{0}\right.$ and $\left.\tilde{\mathrm{t}}_{1} \rightarrow \mathrm{b} \tilde{\boldsymbol{v}} \ell^{+}\right)$have been searched for. The dominant decay mode of the $\tilde{b}_{1}$ is expected to be $\tilde{b}_{1} \rightarrow \mathrm{b} \tilde{\chi}_{1}^{0}$. Since the decay widths of these modes are smaller than the QCD energy scale, the $\tilde{t}_{1}$ and $\tilde{b}_{1}$ produce colorless squark-hadrons before decay. Under the assumption of R-parity conservation, $\tilde{\chi}_{1}^{0}$ and $\tilde{v}$ are invisible in the detector. Thus, $\tilde{\mathrm{t}}_{1} \overline{\tilde{t}}_{1}$ and $\tilde{b}_{1} \overline{\tilde{b}}_{1}$ events are characterised by two acoplanar jets $^{16}$ or two acoplanar jets plus two leptons, with missing energy. The phenomenology of the production and decay of $\tilde{t}_{1}$ and $\tilde{b}_{1}$ is described in Section 2 of Ref. [4].

The CDF Collaboration has reported lower limit values [5] on the $\tilde{\mathfrak{t}}_{1}$ mass of 89 and $110 \mathrm{GeV}$ (95\% C.L.), when the mass difference between $\tilde{\mathrm{t}}_{1}$ and $\tilde{\chi}_{1}^{0}$ is larger than about 40 and $60 \mathrm{GeV}$, respectively. These limits were obtained with the assumption that $\tilde{\mathrm{t}}_{1} \rightarrow \mathrm{c} \tilde{\chi}_{1}^{0}$. Searches at $\mathrm{e}^{+} \mathrm{e}^{-}$colliders are sensitive to smaller mass differences. The first lower limits on the $\tilde{\mathrm{t}}_{1}$ mass were obtained around the $\mathrm{Z}^{0}$ peak (LEP1) assuming $\tilde{\mathrm{t}}_{1} \rightarrow \mathrm{c} \tilde{\chi}_{1}^{0}$ [6]. Using part of the higher energy LEP2 data sample, the $95 \%$ C.L. lower limit for a mass difference larger than $6 \mathrm{GeV}$ was improved to $83 \mathrm{GeV}$ [9]. Several other squark searches at various centre-ofmass energies $(\sqrt{s})$ have also been performed at LEP $[4,7,8,10,11]$.

For the decay mode of $\tilde{t}_{1} \rightarrow$ b $\tilde{v} \ell^{+}$the first lower limit on the $\tilde{\mathrm{t}}_{1}$ mass was obtained at $\sqrt{s}=161 \mathrm{GeV}$ [7], and successive searches were performed at LEP $[4,8-11]$ and the Tevatron. The D0 Collaboration has reported a lower limit [12] on the $\tilde{t}_{1}$ mass of $123 \mathrm{GeV}$ (95\% C.L.), when the mass difference between $\tilde{t}_{1}$ and $\tilde{v}$ is larger than $40 \mathrm{GeV}$ and the branching fraction to each lepton flavour is the same. A search for the fourbody decay mode, $\tilde{\mathrm{t}}_{1} \rightarrow \mathrm{b} \tilde{\chi}_{1}^{0} \mathrm{~W}^{*+}$, where the $\mathrm{W}$ boson is off shell, was recently performed at LEP and no evidence was reported [11].

In 1999 and 2000, the LEP e $\mathrm{e}^{+} \mathrm{e}^{-}$collider at CERN operated at $\sqrt{s}=192-209 \mathrm{GeV}$, and a data sample of

\footnotetext{
15 In this region, $\tilde{\mathrm{t}}_{1} \rightarrow \mathrm{b} \tilde{\chi}_{1}^{0} \mathrm{~W}^{+}$becomes dominant through a virtual chargino. This decay mode has not been studied in this Letter.

16 Two jets are called 'acoplanar' if they not back-to-back with each other in the plane perpendicular to the beam axis.
}

Table 1

List of luminosities and mean values of $\sqrt{s}$ for data collected in 1999 and 2000

\begin{tabular}{ccc}
\hline $\begin{array}{c}s \text { range } \\
(\mathrm{GeV})\end{array}$ & $\begin{array}{c}\text { Luminosity-weighted } \\
\langle\sqrt{s}\rangle(\mathrm{GeV})\end{array}$ & $\begin{array}{c}\text { Luminosity } \\
\left(\mathrm{pb}^{-1}\right)\end{array}$ \\
\hline $190-194$ & 191.6 & 29.1 \\
$194-198$ & 195.5 & 74.0 \\
$198-201$ & 199.5 & 75.4 \\
$201-204$ & 201.6 & 38.3 \\
$204-206$ & 204.9 & 82.0 \\
$>206$ & 206.5 & 138.8 \\
all & 201.7 & 437.6 \\
\hline
\end{tabular}

about $440 \mathrm{pb}^{-1}$ was collected with the OPAL detector. Luminosities and mean values of $\sqrt{s}$ are summarised in Table 1.

In this Letter direct searches for $\tilde{\mathfrak{t}}_{1}$ and $\tilde{b}_{1}$ using this data sample are reported. The limits shown here have been obtained by combining the results obtained at these new centre-of-mass energies with those previously obtained using the OPAL data at lower $\sqrt{s}$ [4,7-9].

\section{The OPAL detector and event simulation}

The OPAL detector, which is described in detail in Ref. [13], is a multipurpose apparatus having nearly complete solid angle coverage. The central detector consists of a silicon strip detector and tracking chambers, providing charged particle tracking for over $96 \%$ of the full solid angle, inside a uniform solenoidal magnetic field of $0.435 \mathrm{~T}$. A lead-glass electromagnetic calorimeter (ECAL) located outside the magnet coil is hermetic in the polar angle range of $|\cos \theta|<0.984$. The magnet return yoke consisting of barrel and endcap sections along with pole tips is instrumented for hadron calorimetry (HCAL) in the region $|\cos \theta|<0.99$. Four layers of muon chambers cover the outside of the hadron calorimeter. Forward detectors (FD), silicon-tungsten calorimeters (SW) and the gamma-catcher detectors (GC) are located in the forward region $(|\cos \theta|>0.98)$ surrounding the beam pipe and provide complete acceptance down to $25 \mathrm{mrad}$.

Monte Carlo simulation of the production and decays of $\tilde{t}_{1}$ and $\tilde{b}_{1}$ were performed following [14]. The 
squark ( $\tilde{\mathrm{q}})$ pairs were generated, and the hadronisation process was subsequently performed to produce colorless $\tilde{\mathrm{q}}$-hadrons and other fragmentation products according to the Lund string fragmentation scheme (JETSET 7.4) $[15,16]$. The parameters for perturbative QCD and fragmentation processes were optimised using hadronic $Z^{0}$ decays measured by OPAL [17]. For the fragmentation of $\tilde{\mathrm{q}}$, the fragmentation function proposed by Peterson et al. [15,18] was used. The $\tilde{\mathrm{q}}$-hadron was formed from a squark and a spectator anti-quark or diquark. For the $\tilde{\mathfrak{t}}_{1}$ decaying into $\mathrm{c} \tilde{\chi}_{1}^{0}$, a colour string was connected between the charm quark and the spectator. The decays $\tilde{b}_{1} \rightarrow \mathrm{b} \tilde{\chi}_{1}^{0}$ and $\tilde{\mathrm{t}}_{1} \rightarrow \mathrm{b} \ell^{+} \tilde{v}$ were simulated in a similar manner. One thousand events were generated at each point of a twodimensional grid of spacing of typically $5 \mathrm{GeV}$ steps in $\left(m_{\tilde{\mathfrak{t}}_{1}}, m_{\tilde{\chi}_{1}^{0}}\right)$ for $\tilde{\mathfrak{t}}_{1} \rightarrow \mathrm{c} \tilde{\chi}_{1}^{0}$, in $\left(m_{\tilde{\mathfrak{t}}_{1}}, m_{\tilde{v}}\right)$ for $\tilde{\mathfrak{t}}_{1} \rightarrow$ $\mathrm{b} \ell^{+} \tilde{v}$ (with equal branching ratios for $\mathrm{e}, \mu$ and $\tau$ ) and $\tilde{\mathrm{t}}_{1} \rightarrow \mathrm{b} \tau^{+} \tilde{v}$, and in $\left(m_{\tilde{\mathrm{b}}_{1}}, m_{\tilde{\chi}_{1}^{0}}\right)$ for $\tilde{\mathrm{b}}_{1} \rightarrow \mathrm{b} \tilde{\chi}_{1}^{0}$. Smaller steps were used for the case of small mass differences $\left(\Delta m=m_{\tilde{t}_{1}}-m_{\tilde{\chi}_{1}^{0}}, m_{\tilde{\mathfrak{t}}_{1}}-m_{\tilde{v}}\right.$ or $\left.m_{\tilde{\mathrm{b}}_{1}}-m_{\tilde{\chi}_{1}^{0}}\right)$. The signal samples were generated at $\sqrt{s}=192,196,200$ and $206 \mathrm{GeV}$.

The background processes were simulated as follows. The KK2f generator [19] was used to simulate multihadronic $(\mathrm{q} \overline{\mathrm{q}}(\gamma))$ events, $\tau^{+} \tau^{-}(\gamma)$, and $\mu^{+} \mu^{-}(\gamma)$ events. Bhabha events, $\mathrm{e}^{+} \mathrm{e}^{-} \rightarrow \mathrm{e}^{+} \mathrm{e}^{-}(\gamma)$, were generated with the BHWIDE program [20]. Twophoton processes are the most important background for the case of small mass differences, since in such cases signal events have small visible energy and small transverse momentum relative to the beam direction. Using the Monte Carlo generators PHOJET [21], PYTHIA [15] and HERWIG [22], hadronic events from various two-photon processes were simulated in which the invariant mass of the photon-photon system $\left(M_{\gamma \gamma}\right)$ was larger than $5.0 \mathrm{GeV}$. Monte Carlo samples for leptonic two-photon processes $\left(\mathrm{e}^{+} \mathrm{e}^{-} \mathrm{e}^{+} \mathrm{e}^{-}\right.$, $\mathrm{e}^{+} \mathrm{e}^{-} \mu^{+} \mu^{-}$and $\mathrm{e}^{+} \mathrm{e}^{-} \tau^{+} \tau^{-}$) were generated with the Vermaseren program [23]. The grc4f [24] and KoralW [25] generators were used for all four-fermion processes except for regions covered by the twophoton simulations. All interference effects of the various diagrams are taken into account in these generators. Four-fermion processes in which at least one of the fermions is a neutrino constitute a serious background at large mass differences. The generated sig- nal and background events were processed through the full simulation of the OPAL detector [26], and the same analysis chain was applied as to the data.

\section{Analysis}

Since the event topologies of $\tilde{\mathrm{t}}_{1} \rightarrow \mathrm{c} \tilde{\chi}_{1}^{0}$ and $\tilde{\mathrm{b}}_{1} \rightarrow$ $\mathrm{b} \tilde{\chi}_{1}^{0}$ are very similar, the same selection criteria were used (Section 3.1, analysis A). In Section 3.2 (analysis $\mathrm{B}$ ), the selection criteria for $\tilde{t}_{1} \rightarrow \mathrm{b} \ell^{+} \tilde{v}$ are discussed. These analyses are the same as those in Ref. [9]. Variables used to make the selections, such as the total visible energy and the total transverse momentum, and jet properties, were calculated as follows. First, the four-momenta of the tracks and those of the ECAL and HCAL clusters not associated with charged tracks were summed. Whenever a calorimeter cluster had associated charged tracks, the expected energy deposited by the tracks was subtracted from the cluster energy to reduce double counting. If the energy of a cluster was smaller than the expected energy deposited by the associated tracks, the cluster energy was not used.

The following three preselections, which are common to analyses A and B, were applied first:

(1) The number of charged tracks was required to be at least four and the visible mass of the event was required to be larger than $3 \mathrm{GeV}$.

(2) The energy deposited had to be less than 5.2 and $5 \mathrm{GeV}$ in each side of the SW, FD and GC detectors, respectively, to reduce the background from two-photon processes.

(3) The visible energy in the region of $|\cos \theta|>0.9$ was required to be less than $10 \%$ of the total visible energy, and the polar angle of the missing momentum direction, $\theta_{\text {miss }}$, was also required to satisfy $\left|\cos \theta_{\text {miss }}\right|<0.9$ to reduce the two-photon and the $\mathrm{q} \overline{\mathrm{q}}(\gamma)$ background.

\subsection{Analysis $A: \tilde{\mathrm{t}}_{1} \rightarrow \mathrm{c} \tilde{\chi}_{1}^{0}$ and $\tilde{\mathrm{b}}_{1} \rightarrow \mathrm{b} \tilde{\chi}_{1}^{0}$}

The experimental signature for $\tilde{\mathrm{t}}_{1} \overline{\tilde{\mathrm{t}}}_{1}\left(\tilde{\mathrm{t}}_{1} \rightarrow \mathrm{c} \tilde{\chi}_{1}^{0}\right)$ events and $\tilde{b}_{1} \overline{\tilde{b}}_{1}$ events is two jets which are not coplanar with the beam axis. The fragmentation functions of $\tilde{\mathrm{t}}_{1}$ and $\tilde{\mathrm{b}}_{1}$ are expected to be hard and the invariant 


\section{OPAL}
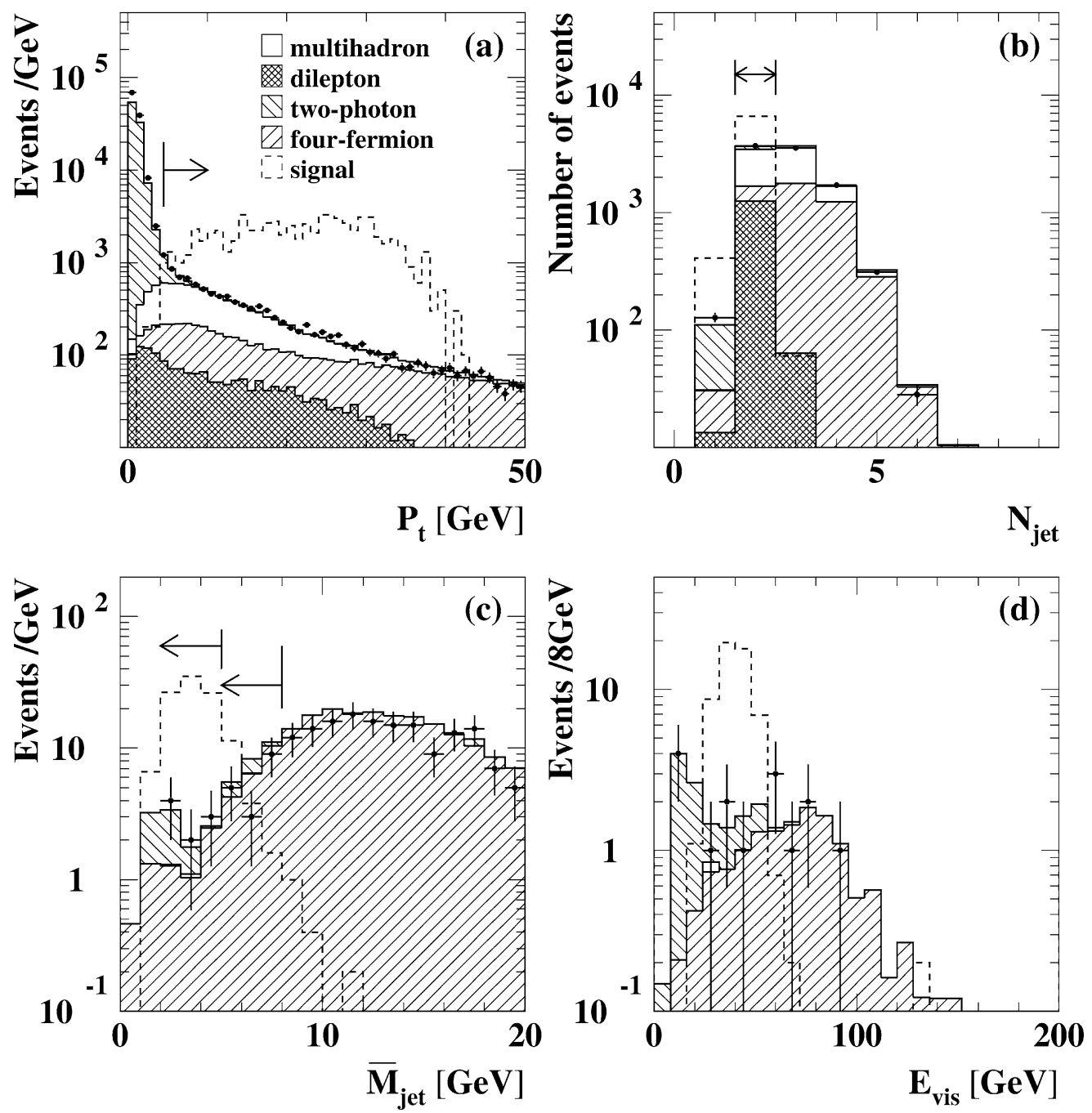

Fig. 1. Distributions of (a) $P_{t}$ before cut (A1), (b) number of reconstructed jets before cut (A2), (c) $\bar{M}_{\text {jet }}$ before cut (A5), (d) $E_{\text {vis }}$ after all selections, for the data, simulated background events and typical $\tilde{\mathrm{t}}_{1} \overline{\tilde{\mathrm{t}}}_{1}$ predictions. In these figures, the distribution of the data is shown as points with error bars. The background processes are as follows: dilepton events (cross-hatched area), two-photon processes (negative slope hatched area), four-fermion processes (positive slope hatched area), and multihadronic events (open area). The arrows show the cut positions. In (c), the left (right) arrow indicates the cut position for $M_{\mathrm{vis}}>65 \mathrm{GeV}\left(M_{\mathrm{vis}}<65 \mathrm{GeV}\right)$. The predictions for $\tilde{\mathrm{t}}_{1} \overline{\tilde{\mathrm{t}}}_{1}$ signals $\left(m_{\tilde{\mathrm{t}}_{1}}=95 \mathrm{GeV}\right.$, $m_{\tilde{\chi}_{1}^{0}}=75 \mathrm{GeV}$ ) are shown by the dashed lines, and the normalisations of the $\tilde{\mathrm{t}}_{1} \overline{\tilde{\mathrm{t}}}_{1}$ predictions are arbitrary.

mass of the charm (or bottom) quark and the spectator quark is small, therefore the jets are expected to be narrow and have low invariant masses. The following five selections were applied.

(A1) Events from two-photon processes were largely removed by demanding that the missing transverse momentum, $P_{t}$, is greater than $4.5 \mathrm{GeV}$. Fig. 1(a) shows the distribution of $P_{t}$ after the preselection.

(A2) The number of reconstructed jets was required to be exactly two. Jets were reconstructed using the Durham algorithm [27] with the jet resolution parameter of $y_{\text {cut }}=0.005\left(E_{\mathrm{vis}} / \sqrt{s}\right)^{-1}$, where $E_{\mathrm{vis}}$ 
is the total visible energy. This $E_{\mathrm{vis}}$-dependent $y_{\text {cut }}$ parameter was necessary for good jet reconstruction over a wide range of $m_{\tilde{\mathfrak{t}}_{1}}, m_{\tilde{\mathrm{b}}_{1}}$ and $m_{\tilde{\chi}_{1}^{0}}$, and the distribution of the number of reconstructed jets is shown in Fig. 1(b). Both reconstructed jets were required to contain at least two charged particles to reduce the $\tau^{+} \tau^{-}$background.

(A3) The acoplanarity angle, $\phi_{\text {acop }}$, is defined as $\pi$ minus the azimuthal opening angle between the directions of the two reconstructed jets. To ensure the reliability of the calculation of $\phi_{\text {acop }}$, both jet axes were required to have a polar angle satisfying $\left|\cos \theta_{\text {jet }}\right|<0.95$. The value of $\phi_{\text {acop }}$ was required to be larger than $20^{\circ}$.

(A4) Softness, $\mathcal{S}$, was defined as $\left(\frac{M_{1}}{E_{1}}+\frac{M_{2}}{E_{2}}\right)$, where $M_{1}$ and $M_{2}$ are the invariant masses of the two reconstructed jets, and $E_{1}$ and $E_{2}$ are the energies of the jets. The signal events have low values of $\mathcal{S}$, whereas two-photon events which pass the acoplanarity cut have relatively large values [8]. It was required that $1.5 \times \mathcal{S}<\left(P_{t}-4.5\right)$, where $P_{t}$ is given in units of $\mathrm{GeV}$.

(A5) The arithmetic mean of the invariant masses of the jets, $\bar{M}_{\text {jet }}$, was required to be smaller than $8 \mathrm{GeV}$. When the invariant mass of the event, $M_{\text {vis }}$, was larger than $65 \mathrm{GeV}$, a harder cut, $\bar{M}_{\text {jet }}<5 \mathrm{GeV}$, was applied to reduce background from Wev events. Fig. 1(c) shows the $\bar{M}_{\text {jet }}$ distributions for data, the simulated background processes and typical $\tilde{\mathrm{t}}_{1} \overline{\tilde{t}}_{1}$ events. As shown in this figure, jets from $\tilde{\mathrm{t}}_{1}$ are expected to have low invariant masses.

The numbers of events remaining after each cut are listed in Table 2. The table also shows the corresponding numbers of simulated events for background processes. After all cuts, 13 events were observed in the data, which is consistent with the expected number of background events of $19.8 \pm 2.2$. Fig. 1(d) shows the $E_{\text {vis }}$ distribution after all selections were applied.

The efficiencies for both $\tilde{\mathrm{t}}_{1} \overline{\tilde{\mathrm{t}}}_{1}$ and $\tilde{\mathrm{b}}_{1} \overline{\tilde{\mathrm{b}}}_{1}$ events are $30-60 \%$ if the mass difference between $\tilde{\mathfrak{t}}_{1}\left(\tilde{\mathrm{b}}_{1}\right)$ and $\tilde{\chi}_{1}^{0}$ is larger than $10 \mathrm{GeV}$. A modest efficiency of about $20 \%$ is obtained for a mass difference of $5 \mathrm{GeV}$ for $\tilde{\mathfrak{t}}_{1} \overline{\tilde{t}}_{1}$ events. An additional loss of $3 \%$ (relative) arises from beam-related background in SW, FD and $\mathrm{GC}$, which was estimated using random beam crossing events.

\subsection{Analysis $B: \tilde{\mathrm{t}}_{1} \rightarrow \mathrm{b} \ell \tilde{v}$}

The experimental signature for $\tilde{\mathrm{t}}_{1} \overline{\tilde{t}}_{1}\left(\tilde{\mathrm{t}}_{1} \rightarrow \mathrm{b} \ell \tilde{v}\right)$ events is two acoplanar jets plus two leptons with missing transverse momentum. The momenta of the leptons and the missing transverse momentum depend strongly on the mass difference between $\tilde{\mathrm{t}}_{1}$ and $\tilde{v}$. To obtain optimal performance, two sets of selection criteria (analyses B-L and B-H) were applied for small and large mass differences, respectively.

The numbers of events remaining after each cut are listed in Tables 3 and 4. The tables also show the corresponding numbers for the simulated background processes.

\subsubsection{Small mass difference case}

For the case of a small mass difference $(\Delta m \leqslant$ $10 \mathrm{GeV}$ ), the following four selection criteria were applied. Lepton identification was not used in this analysis.

(B-L1) The event missing transverse momentum, $P_{t}$, was required to be greater than $5 \mathrm{GeV}$.

(B-L2) The number of charged tracks was required to be at least six, and the number of reconstructed jets was required to be at least four, since the signal would contain two hadronic jets plus two isolated leptons. Jets were reconstructed using the Durham algorithm [27] with the jet resolution parameter $y_{\text {cut }}=0.004$. Fig. 2(a) shows the distribution of the number of reconstructed jets for the data, the simulated background processes and typical $\tilde{\mathrm{t}}_{1} \overline{\tilde{\mathrm{t}}}_{1}$ events.

(B-L3) To examine the acoplanarity of the remaining events, the whole event was reconstructed as two jets using the Durham algorithm. To ensure a good measurement of the acoplanarity angle, $\left|\cos \theta_{\text {jet }}\right|<$ 0.95 was required for both reconstructed jets. Finally, the acoplanarity angle, $\phi_{\text {acop }}$, between these two jets was required to be greater than $15^{\circ}$. Fig. 2(b) shows the $\phi_{\text {acop }}$ distributions.

(B-L4) The total visible energy, $E_{\mathrm{vis}}$, was required to be smaller than $60 \mathrm{GeV}$ to reject four-fermion events. As shown in Fig. 2(c), a large fraction of fourfermion events are removed.

Five events were observed in the data after all the cuts, which is consistent with the number of expected background events $(5.0 \pm 1.4)$, mainly from twophoton processes. The detection efficiencies are 30 
Table 2

Numbers of events remaining after each cut for various background processes are compared with data for analysis A. The simulated background processes were normalised to the integrated luminosity of the data. The errors due to Monte Carlo statistics are also shown. Efficiencies for three simulated event samples $(\sqrt{s}=206 \mathrm{GeV})$ of $\tilde{\mathrm{t}}_{1} \overline{\tilde{\mathrm{t}}}_{1}$ and $\tilde{\mathrm{b}}_{1} \overline{\tilde{\mathrm{b}}}_{1}$ are also given

\begin{tabular}{|c|c|c|c|c|c|c|c|c|c|}
\hline & Data & $\begin{array}{l}\text { Total } \\
\text { bkg. }\end{array}$ & $\mathrm{q} \overline{\mathrm{q}}(\gamma)$ & $\ell^{+} \ell^{-}(\gamma)$ & $\begin{array}{c}\text { Two- } \\
\text { photon }\end{array}$ & 4-f & \multicolumn{3}{|c|}{$\begin{array}{l}\text { Efficiency for } \\
\tilde{\mathfrak{t}}_{1} \overline{\tilde{t}}_{1} \text { and } \tilde{\mathrm{b}}_{1} \overline{\tilde{b}}_{1}\end{array}$} \\
\hline$m_{\tilde{\mathfrak{t}}_{1}}(\mathrm{GeV})$ & & & & & & & 95 & 95 & - \\
\hline$m_{\tilde{\mathrm{b}}_{1}}(\mathrm{GeV})$ & & & & & & & - & - & 95 \\
\hline$m_{\tilde{\chi}_{1}^{0}}(\mathrm{GeV})$ & & & & & & & 90 & 75 & 75 \\
\hline cut (A1) & 9279 & 9429 & 4147 & 1318 & 313 & 3650 & 0.40 & 0.70 & 0.70 \\
\hline cut (A2) & 2189 & 2243 & 1719 & 92.0 & 112 & 319 & 0.25 & 0.62 & 0.65 \\
\hline cut (A3) & 205 & 241 & 0.35 & 0.52 & 20.6 & 220 & 0.24 & 0.56 & 0.60 \\
\hline cut (A4) & 198 & 229 & 0.32 & 0.52 & 9.0 & 220 & 0.18 & 0.56 & 0.59 \\
\hline cut (A5) & 13 & $\begin{array}{r}19.8 \\
( \pm 2.2)\end{array}$ & $\begin{array}{r}0.25 \\
( \pm 0.13)\end{array}$ & $\begin{array}{r}0.25 \\
( \pm 0.08)\end{array}$ & $\begin{array}{r}7.7 \\
( \pm 2.1)\end{array}$ & $\begin{array}{r}11.6 \\
( \pm 0.6)\end{array}$ & 0.18 & 0.55 & 0.58 \\
\hline
\end{tabular}

Table 3

Numbers of events remaining after each cut for various background processes are compared with data for analysis B-L. The simulated background processes were normalised to the integrated luminosity of the data. The errors due to Monte Carlo statistics are also shown. Efficiencies for two simulated samples of $\tilde{\mathrm{t}}_{1} \overline{\tilde{t}}_{1}$ are also given. In these samples, produced at $\sqrt{s}=206 \mathrm{GeV}$, the branching fractions to each lepton flavour are assumed to be the same

\begin{tabular}{|c|c|c|c|c|c|c|c|c|}
\hline & Data & $\begin{array}{l}\text { Total } \\
\text { bkg. }\end{array}$ & $\mathrm{q} \overline{\mathrm{q}}(\gamma)$ & $\ell^{+} \ell^{-}(\gamma)$ & $\begin{array}{c}\text { Two- } \\
\text { photon }\end{array}$ & $4-f$ & \multicolumn{2}{|c|}{$\begin{array}{l}\text { Efficiency } \\
\text { for } \tilde{\mathfrak{t}}_{1} \overline{\tilde{t}}_{1}\end{array}$} \\
\hline$m_{\tilde{t}_{1}}(\mathrm{GeV})$ & & & & & & & 95 & 95 \\
\hline$m_{\tilde{v}}(\mathrm{GeV})$ & & & & & & & 88 & 85 \\
\hline cut (B-L1) & 8922 & 8983 & 3916 & 1274 & 230 & 3563 & 0.14 & 0.47 \\
\hline cut (B-L2) & 2259 & 2252 & 560 & 0.13 & 15.6 & 1676 & 0.11 & 0.42 \\
\hline cut (B-L3) & 513 & 496 & 17.6 & 0.02 & 3.73 & 474 & 0.11 & 0.39 \\
\hline cut (B-L4) & 5 & $\begin{array}{r}5.02 \\
( \pm 1.36)\end{array}$ & $\begin{array}{r}0.17 \\
( \pm 0.09)\end{array}$ & 0.00 & $\begin{array}{r}3.62 \\
( \pm 1.34)\end{array}$ & $\begin{array}{r}1.22 \\
( \pm 0.19)\end{array}$ & 0.11 & 0.39 \\
\hline
\end{tabular}

Table 4

Numbers of events remaining after each cut for various background processes are compared with data for analysis B-H. The simulated background processes were normalised to the integrated luminosity of the data. The errors due to Monte Carlo statistics are also shown. Efficiencies for three simulated samples of $\tilde{\mathrm{t}}_{1} \overline{\tilde{\mathrm{t}}}_{1}$ are also given. In these samples, produced at $\sqrt{s}=206 \mathrm{GeV}$, the branching fractions to each lepton flavour are assumed to be the same

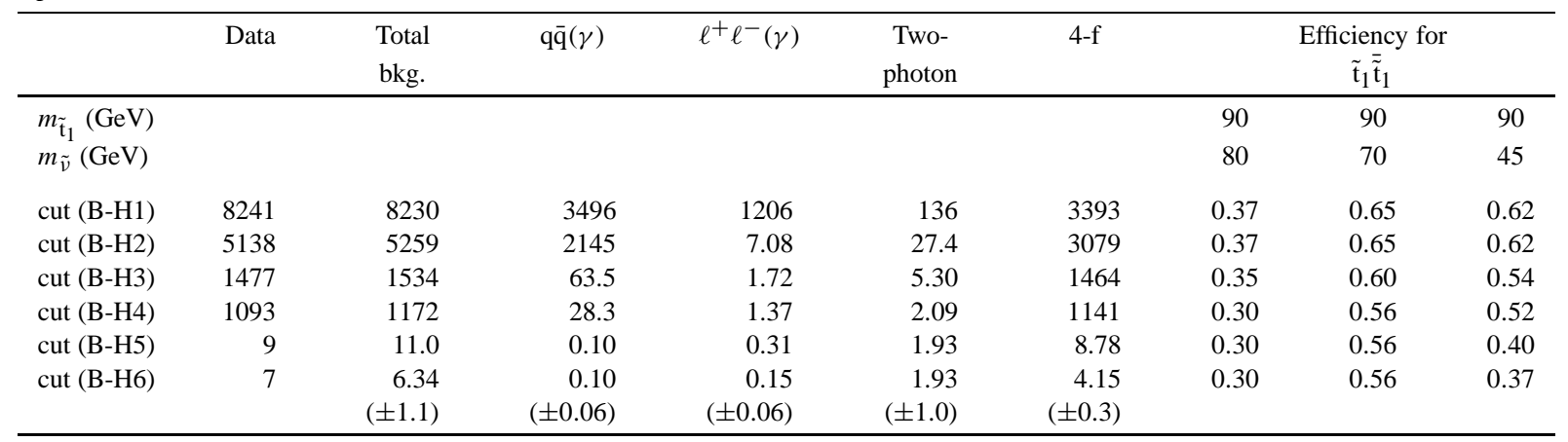




\section{OPAL}
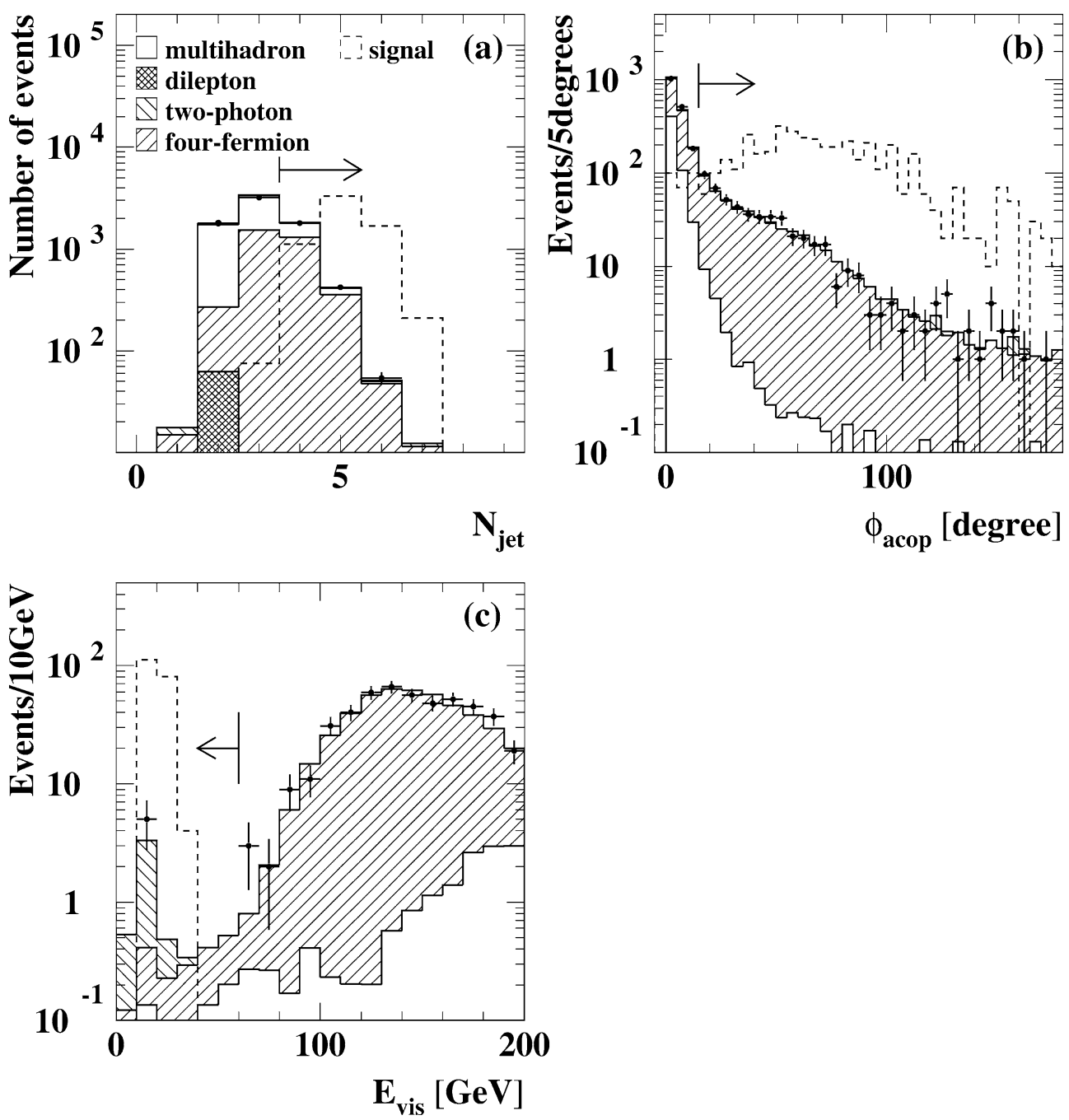

Fig. 2. Distributions of (a) number of reconstructed jets after cut (B-L1), (b) $\phi_{\text {acop }}$ before cut (B-L3), (c) $E_{\text {vis }}$ before cut (B-L4). The conventions for the various histograms are the same as in Fig. 1. The $\tilde{\mathfrak{t}}_{1} \overline{\tilde{t}}_{1}$ predictions show the cases of $\left(m_{\tilde{\mathfrak{t}}_{1}}, m_{\tilde{\chi}_{1}^{0}}\right)=(95 \mathrm{GeV}, 85 \mathrm{GeV})$.

$40 \%$ if the mass difference between $\tilde{\mathrm{t}}_{1}$ and $\tilde{v}$ is $10 \mathrm{GeV}$, and if the branching fraction to each lepton flavour is the same. Even if the branching fraction into $b \tau^{+} \tilde{\nu}_{\tau}$ is $100 \%$, the efficiencies are $25-35 \%$.

\subsubsection{Large mass difference case}

The selection criteria for a large mass difference $(\Delta m>10 \mathrm{GeV})$ are as follows:
(B-H1) The event missing transverse momentum, $P_{t}$, was required to be greater than $6 \mathrm{GeV}$.

(B-H2) The number of charged tracks was required to be at least six, and the number of reconstructed jets was required to be at least three. Jets were reconstructed with the same jet resolution parameter $\left(y_{\text {cut }}=0.004\right)$ as in $(\mathrm{B}-\mathrm{L} 2)$.

(B-H3) The same selection as (B-L3) was applied on the $\phi_{\text {acop }}$ variable to reject $\mathrm{q} \overline{\mathrm{q}}(\gamma)$ events. 

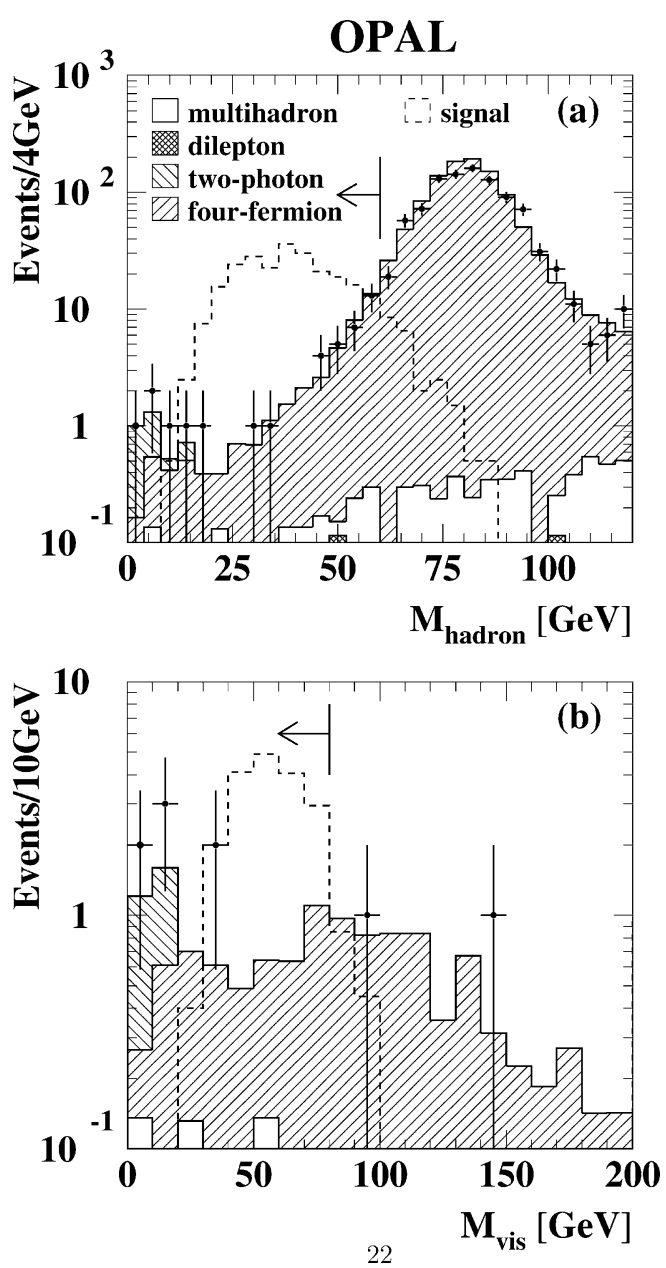

Fig. 3. Distributions of (a) invariant mass excluding the most energetic lepton before cut (B-H5), (b) $M_{\text {vis }}$ before cut (B-H6). The conventions for the various histograms are the same as in Fig. 1. The $\tilde{\mathfrak{t}}_{1} \overline{\tilde{t}}_{1}$ predictions show the cases of $\left(m_{\tilde{\mathrm{t}}_{1}}\right.$, $\left.m_{\tilde{\chi}_{1}^{0}}\right)=(95 \mathrm{GeV}, 47.5 \mathrm{GeV})$.

(B-H4) A candidate event was required to contain at least one lepton, since a signal event would contain two isolated leptons. The selection criteria for leptons are given in Ref. [8].

(B-H5) The invariant mass of the event excluding the most energetic lepton, $M_{\text {hadron, }}$ was required to be smaller than $60 \mathrm{GeV}$ in order to reject $\mathrm{W}^{+} \mathrm{W}^{-} \rightarrow$ $\nu \ell \mathrm{q} \overline{\mathrm{q}}^{\prime}$ events. As shown in Fig. 3(a), a large fraction of four-fermion events was rejected using this requirement. Furthermore the invariant mass excluding all identified leptons was required to be smaller than $40 \mathrm{GeV}$.

(B-H6) Finally, the visible mass of the event, $M_{\mathrm{vis}}$, must be smaller than $80 \mathrm{GeV}$ to reduce $\mathrm{W}^{+} \mathrm{W}^{-}$ background events in which one of $\mathrm{W}^{ \pm}$'s decays into $\tau \nu$ and the other into $q \bar{q}^{\prime}(\mathrm{g})$. If one jet from $\mathrm{q} \overline{\mathrm{q}}^{\prime}(\mathrm{g})$ was misidentified as a tau lepton, this event could pass through the previous cut (B-H5). Fig. 3(b) shows the $M_{\text {vis }}$ distributions.

Seven candidate events were observed in the data, which is consistent with the number of expected background events $(6.3 \pm 1.1)$. The dominant background arises from four-fermion processes. The detection efficiencies are $30-60 \%$, if the mass difference between the $\tilde{t}_{1}$ and $\tilde{v}$ is $10 \mathrm{GeV}$, and if the $\tilde{v}$ is heavier than $30 \mathrm{GeV}$. The detection efficiencies for $\tilde{\mathfrak{t}}_{1}$ were found to be slightly smaller for the case where it decays purely into $b \tau^{+} \tilde{\nu}_{\tau}$ than for the case where the branching fraction to each lepton flavour is assumed to be the same.

\section{Results}

The observed number of candidate events in each case is consistent with the expected number of background processes. Since no evidence for $\tilde{\mathrm{t}}_{1} \overline{\tilde{t}}_{1}$ and $\tilde{\mathrm{b}}_{1} \overline{\tilde{b}}_{1}$ pair-production has been observed, lower limits on $m_{\tilde{\mathfrak{t}}_{1}}$ and $m_{\tilde{\mathrm{b}}_{1}}$ are calculated. The results shown here have been obtained by combining the results obtained at these new centre-of-mass energies with those previously obtained using the OPAL data at lower $\sqrt{s}$ [4,7-9].

The systematic errors on the expected number of signal and background events were estimated in the same manner as in the previous paper [8]. The main sources of systematic errors on the signal are uncertainties in the $\tilde{\mathrm{t}}_{1}$ and $\tilde{\mathrm{b}}_{1}$ fragmentation $(5-15 \%)$ and in Fermi motion of the spectator quark (3-10\%). The main sources of systematic errors on the background are uncertainties in the generation of four-fermion processes $(5 \%)$. The background from four-fermion processes evaluated with the grc $4 \mathrm{f}$ and KoralW generators agreed within the statistical error, but the small difference was conservatively taken as a systematic error. The limited statistics of the two-photon Monte Carlo samples also give rise to a sizable systematic error. Detailed descriptions are given in Ref. [8]. Sys- 

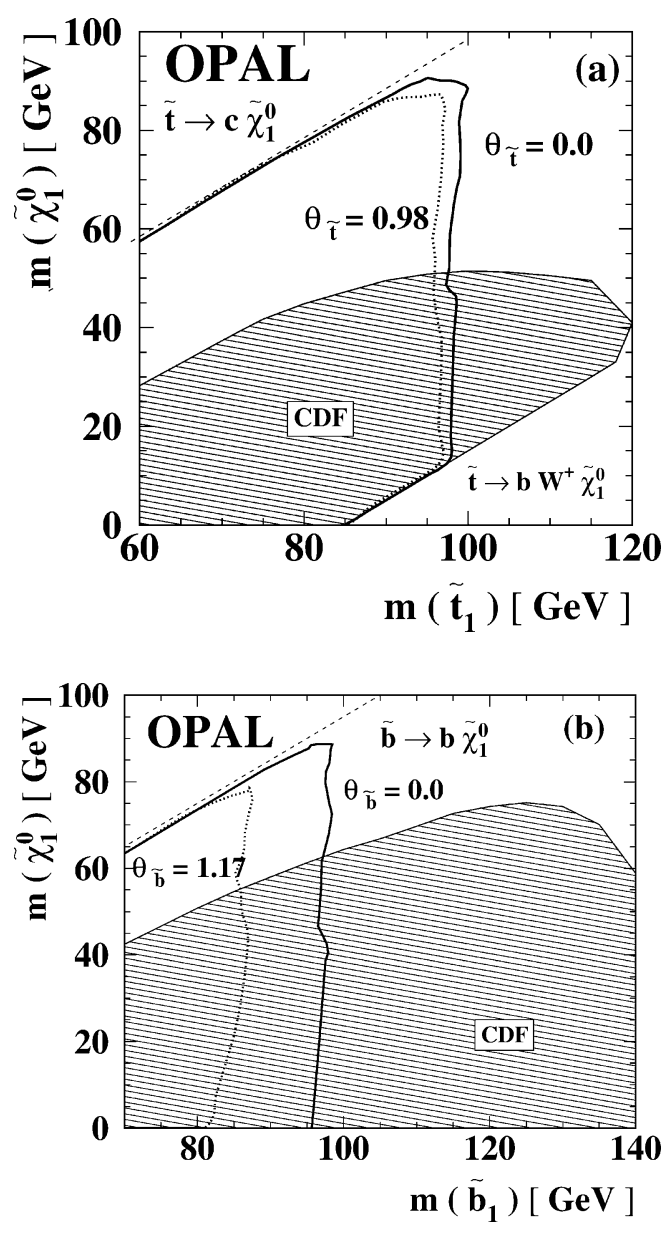

Fig. 4. (a) The $95 \%$ C.L. excluded regions in the $\left(m_{\tilde{t}_{1}}, m_{\tilde{\chi}_{1}^{0}}\right)$ plane assuming that $\tilde{\mathrm{t}}_{1}$ decays into $\mathrm{c} \tilde{\chi}_{1}^{0}$. The solid line shows the limit for zero mixing angle of $\tilde{t}_{1}$, and the dotted line shows the limit for a mixing angle of $0.98 \mathrm{rad}\left(\tilde{\mathrm{t}}_{1}\right.$ decouples from the $Z^{0}$ boson). The dash-dotted straight line shows the kinematic limit for the $\tilde{\mathrm{t}}_{1} \rightarrow \mathrm{c} \tilde{\chi}_{1}^{0}$ decay. In the triangular region of $m_{\tilde{\mathrm{t}}_{1}}-m_{\tilde{\chi}_{1}^{0}}>m_{\mathrm{W}^{ \pm}}+m_{\mathrm{b}}$, the decay $\tilde{\mathrm{t}}_{1} \rightarrow \mathrm{b} \tilde{\chi}_{1}^{0} \mathrm{~W}^{+}$(on shell) through a virtual chargino becomes dominant. This region is not excluded. (b) The $95 \%$ C.L. excluded regions in the $\left(m_{\tilde{\mathrm{b}}_{1}}, m_{\tilde{\chi}_{1}^{0}}\right)$ plane, assuming that $\tilde{\mathrm{b}}_{1}$ decays into $\mathrm{b} \tilde{\chi}_{1}^{0}$. The solid line shows the limit where the mixing angle of $\tilde{b}_{1}$ is assumed to be zero, and the dotted line shows the limits for a mixing angle of $1.17 \mathrm{rad}\left(\tilde{b}_{1}\right.$ decouples from the $Z^{0}$ boson). The singly-hatched regions in (a) and (b) are excluded by the CDF Collaboration [5].

tematic errors are taken into account when calculating limits [28].

Fig. 4(a) shows the 95\% C.L. excluded regions in the $\left(m_{\tilde{\mathfrak{t}}_{1}}, m_{\tilde{\chi}_{1}^{0}}\right)$ plane for $\tilde{\mathfrak{t}}_{1} \rightarrow \mathrm{c} \tilde{\chi}_{1}^{0}$. In this figure there
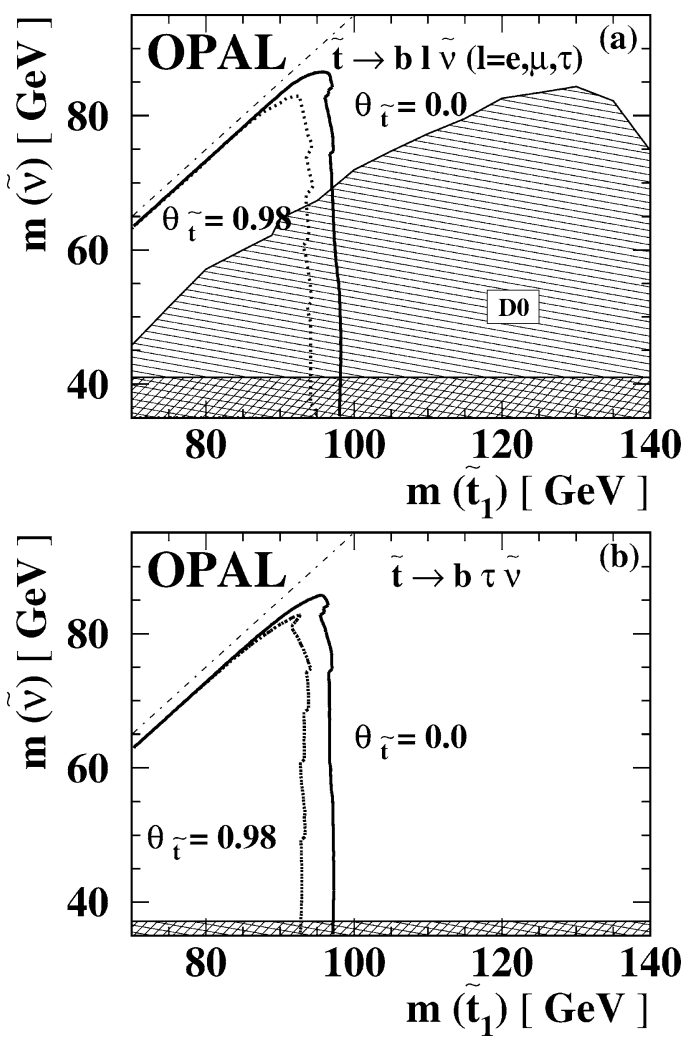

Fig. 5. The $95 \%$ C.L. excluded regions in the $\left(m_{\tilde{t}_{1}}, m_{\tilde{v}}\right)$ plane assuming that the $\tilde{t}_{1}$ decays into $b \ell \tilde{v}$; (a) the branching fraction to each lepton flavour is the same; (b) $\tilde{t}_{1}$ always decays into $b \tau \tilde{v}_{\tau}$. The solid lines show the limits where the mixing angle of $\tilde{t}_{1}$ is assumed to be zero, and the dotted lines show the limits for a mixing angle of $0.98 \mathrm{rad}$ (decoupling case). The cross-hatched region has been excluded by measurements of the $Z^{0}$ invisible decay width at LEP1 [29], and the dash-dotted diagonal line shows the kinematic limit for the $\tilde{t}_{1} \rightarrow b \ell \tilde{v}$ decay. The singly-hatched region in (a) is excluded by the D0 Collaboration [12].

is a triangular region of $m_{\tilde{\mathfrak{t}}_{1}}-m_{\tilde{\chi}_{1}^{0}}>m_{\mathrm{W}^{ \pm}}+m_{\mathrm{b}}$, in which $\tilde{\mathrm{t}}_{1} \rightarrow \mathrm{b} \tilde{\chi}_{1}^{0} \mathrm{~W}^{+}$(on shell) through a virtual chargino becomes dominant even if the chargino is heavy. This region is not excluded.

Figs. 5(a) and (b) show the 95\% C.L. excluded regions in the $\left(m_{\tilde{\mathrm{t}}_{1}}, m_{\tilde{v}}\right)$ plane for $\tilde{\mathrm{t}}_{1} \rightarrow \mathrm{b} \ell \tilde{v}(\ell=\mathrm{e}, \mu, \tau)$ and $\tilde{\mathrm{t}}_{1} \rightarrow \mathrm{b} \tau^{+} \tilde{v}_{\tau}$, respectively. The branching fraction to each lepton flavour $\ell^{+}$depends on the composition of the lightest chargino [4]. As the chargino becomes more Higgsino-like, the branching fraction into $\mathrm{b} \tau^{+} \tilde{\nu}_{\tau}$ becomes large. In the limit that the chargino is a pure Wino state, the branching fraction to each lepton 
Table 5

The excluded $m_{\tilde{t}_{1}}$ region at $95 \%$ C.L. $\left(\Delta m=m_{\tilde{\mathfrak{t}}_{1}}-m_{\tilde{\chi}_{1}^{0}}\right.$ or $\left.m_{\tilde{\mathfrak{t}}_{1}}-m_{\tilde{v}}\right)$

\begin{tabular}{|c|c|c|c|c|}
\hline \multirow[b]{3}{*}{$\theta_{\tilde{\mathrm{t}}}(\mathrm{rad})$} & \multicolumn{4}{|c|}{ Lower limit on $m_{\tilde{t}_{1}}(\mathrm{GeV})$} \\
\hline & \multicolumn{2}{|c|}{$\tilde{\mathrm{t}}_{1} \rightarrow \mathrm{c} \tilde{\chi}_{1}^{0}$} & \multirow{2}{*}{$\begin{array}{c}\tilde{\mathrm{t}}_{1} \rightarrow \mathrm{b} \ell \tilde{v} \\
\ell=\mathrm{e}, \mu, \tau \\
\Delta m \geqslant 10 \mathrm{GeV}\end{array}$} & \multirow{2}{*}{$\begin{array}{c}\tilde{\mathrm{t}}_{1} \rightarrow \mathrm{b} \tau \tilde{\nu}_{\tau} \\
\mathrm{Br}=100 \% \\
\Delta m \geqslant 10 \mathrm{GeV}\end{array}$} \\
\hline & $\Delta m \geqslant 5 \mathrm{GeV}$ & $\Delta m \geqslant 10 \mathrm{GeV}$ & & \\
\hline 0.0 & 95.2 & 97.6 & 96.0 & 95.5 \\
\hline 0.98 & 91.4 & 95.7 & 92.6 & 91.5 \\
\hline
\end{tabular}

Table 6

The excluded $m_{\tilde{\mathrm{b}}_{1}}$ region at $95 \%$ C.L. $\left(\Delta m=m_{\tilde{\mathrm{b}}_{1}}-m_{\tilde{\chi}_{1}^{0}}\right)$

\begin{tabular}{lcc}
\hline & \multicolumn{2}{c}{ Lower limit on $m_{\tilde{\mathrm{b}}_{1}}(\mathrm{GeV})\left(\tilde{\mathrm{b}}_{1} \rightarrow \mathrm{b} \tilde{\chi}_{1}^{0}\right)$} \\
\cline { 2 - 3 }$\theta_{\tilde{\mathrm{b}}}(\mathrm{rad})$ & $\Delta m \geqslant 7 \mathrm{GeV}$ & $\Delta m \geqslant 10 \mathrm{GeV}$ \\
\hline 0.0 & 93.5 & 96.9 \\
1.17 & 82.6 & 85.1 \\
\hline
\end{tabular}

flavour is the same. Two extreme cases in which the branching fraction to each lepton flavour is the same, or the branching fraction into $b \tau^{+} \tilde{\nu}_{\tau}$ is $100 \%$, were considered here.

The 95\% C.L. mass bounds of $\tilde{\mathfrak{t}}_{1}$ are listed in Table 5 for two values of $\theta_{\tilde{\mathfrak{t}}}$. Assuming that $\tilde{\mathfrak{t}}_{1}$ decays into $\mathrm{c} \tilde{\chi}_{1}^{0}$, and the mass difference between $\tilde{\mathrm{t}}_{1}$ and $\tilde{\chi}_{1}^{0}$ is greater than $10 \mathrm{GeV}, \tilde{\mathfrak{t}}_{1}$ is found to be heavier than $97.6 \mathrm{GeV}$ for $\theta_{\tilde{\mathrm{t}}}=0.0$. A lower limit of $95.7 \mathrm{GeV}$ is obtained even if $\tilde{\mathfrak{t}}_{1}$ decouples from the $Z^{0}$ boson $\left(\theta_{\tilde{\mathfrak{t}}}=0.98 \mathrm{rad}\right)$, which approximately minimizes the cross-section. When $\tilde{\mathrm{t}}_{1}$ decays into $\mathrm{b} \ell \tilde{v}$, the lower limit on $m_{\tilde{\mathfrak{t}}_{1}}$ is $96.0 \mathrm{GeV}$ for the zero mixing angle case, assuming that the mass difference between $\tilde{t}_{1}$ and $\tilde{v}$ is greater than $10 \mathrm{GeV}$ and that the branching fraction to each lepton flavour is the same.

The $95 \%$ C.L. excluded regions in the $\left(m_{\tilde{\mathrm{b}}_{1}}, m_{\tilde{\chi}_{1}^{0}}\right)$ plane are shown in Fig. 4(b) for two cases $\theta_{\tilde{b}}=0$ and $1.17 \mathrm{rad}$. The numerical mass bounds are listed in Table 6 for two values of $\theta_{\tilde{b}}$. The lower limit on the $\tilde{\mathrm{b}}_{1}$-mass is found to be $96.9 \mathrm{GeV}$, if $\Delta m$ is greater than $10 \mathrm{GeV}$ and $\theta_{\tilde{\mathrm{b}}}=0.0$. If the $\tilde{\mathrm{b}}_{1}$ decouples from the $\mathrm{Z}^{0}$ boson $\left(\theta_{\tilde{\mathrm{b}}}=1.17 \mathrm{rad}\right)$, the lower limit is $85.1 \mathrm{GeV}$. Since the electromagnetic charge of $\tilde{b}_{1}$ is half that of $\tilde{t}_{1}$, the coupling between $\gamma$ and $\tilde{b}_{1}$ is weaker than between $\gamma$ and $\tilde{t}_{1}$. Therefore, the production crosssection of $\tilde{b}_{1} \overline{\tilde{b}}_{1}$ is strongly suppressed when the $\tilde{b}_{1}$ decouples from the $\mathrm{Z}^{0}$ boson.

\section{Summary and conclusion}

A data sample of $437.6 \mathrm{pb}^{-1}$ collected using the OPAL detector at $\sqrt{s}=192-209 \mathrm{GeV}$ has been analysed to search for pair production of the scalar top quark and the scalar bottom quark predicted by supersymmetric theories, assuming R-parity conservation. No evidence was found above the background level expected from the Standard Model.

The 95\% C.L. lower limit on the scalar top quark mass is $97.6 \mathrm{GeV}$ if the mixing angle of the scalar top quark is zero. Even if the $\tilde{\mathrm{t}}_{1}$ decouples from the $\mathrm{Z}^{0}$ boson, a lower limit of $95.7 \mathrm{GeV}$ is obtained. These limits were estimated assuming that the scalar top quark decays into a charm quark and the lightest neutralino and that the mass difference between the scalar top and the lightest neutralino is larger than $10 \mathrm{GeV}$.

Assuming a relatively light scalar neutrino $\left(m_{\tilde{v}} \leqslant\right.$ $m_{\tilde{\mathrm{t}}_{1}}-m_{\mathrm{b}}$ ), the complementary decay mode, in which the scalar top quark decays into a bottom quark, a charged lepton and a scalar neutrino, has also been studied. If the mass difference between the scalar top quark and the scalar neutrino is greater than $10 \mathrm{GeV}$ and if the mixing angle of the scalar top quark is zero, the $95 \%$ C.L. lower limit on the scalar top quark mass is $96.0 \mathrm{GeV}$. This limit is obtained assuming that the branching fraction to each lepton flavour is the same. If the branching fraction to the tau lepton is $100 \%$, a lower limit of $95.5 \mathrm{GeV}$ is obtained.

The lower limit on the light scalar bottom quark mass is found to be $96.9 \mathrm{GeV}$, assuming that the mass difference between the scalar bottom quark and the lightest neutralino is greater than $10 \mathrm{GeV}$ and that the mixing angle of the scalar bottom quark is zero. When the scalar bottom quark decouples from the $\mathrm{Z}^{0}$ boson, a lower limit of $85.1 \mathrm{GeV}$ is obtained. These limits are significantly improved with respect to the previous 
OPAL results [9], and are the best limits published to date.

\section{Acknowledgements}

We particularly wish to thank the SL Division for the efficient operation of the LEP accelerator at all energies and for their close cooperation with our experimental group. In addition to the support staff at our own institutions we are pleased to acknowledge the Department of Energy, USA, National Science Foundation, USA, Particle Physics and Astronomy Research Council, UK, Natural Sciences and Engineering Research Council, Canada, Israel Science Foundation, administered by the Israel Academy of Science and Humanities, Benoziyo Center for High Energy Physics, Japanese Ministry of Education, Culture, Sports, Science and Technology (MEXT) and a grant under the MEXT International Science Research Program, Japanese Society for the Promotion of Science (JSPS), German Israeli Bi-national Science Foundation (GIF), Bundesministerium für Bildung und Forschung, Germany, National Research Council of Canada, Hungarian Foundation for Scientific Research, OTKA T-029328, and T-038240, Fund for Scientific Research, Flanders, F.W.O.-Vlaanderen, Belgium.

\section{References}

[1] M. Drees, K. Hikasa, Phys. Lett. B 252 (1990) 127; K. Hikasa, M. Kobayashi, Phys. Rev. D 36 (1987) 724.

[2] A. Bartl, W. Majerotto, W. Porod, Z. Phys. C 64 (1994) 499.

[3] P. Fayet, Phys. Lett. B 69 (1977) 489.

[4] OPAL Collaboration, K. Ackerstaff, et al., Z. Phys. C 75 (1997) 409.

[5] CDF Collaboration, T. Affolderi, et al., Phys. Rev. Lett. 84 (2000) 5704.

[6] OPAL Collaboration, R. Akers, et al., Phys. Lett. B 337 (1994) 207.

[7] OPAL Collaboration, K. Ackerstaff, et al., Phys. Lett. B 389 (1996) 197.

[8] OPAL Collaboration, K. Ackerstaff, et al., Eur. Phys. J. C 6 (1999) 225
[9] OPAL Collaboration, G. Abbiendi, et al., Phys. Lett. B 456 (1999) 95.

[10] ALEPH Collaboration, R. Barate, et al., Phys. Lett. B 413 (1997) 431;

ALEPH Collaboration, R. Barate, et al., Phys. Lett. B 434 (1998) 189;

ALEPH Collaboration, R. Barate, et al., Phys. Lett. B 499 (2001) 67;

ALEPH Collaboration, R. Barate, et al., Phys. Lett. B 488 (2000) 234;

DELPHI Collaboration, P. Abreu, et al., Eur. Phys. J. C 6 (1999) 385;

DELPHI Collaboration, P. Abreu, et al., Phys. Lett. B 496 (2000) 59;

L3 Collaboration, M. Acciarri, et al., Phys. Lett. B 445 (1999) 428;

L3 Collaboration, M. Acciarri, et al., Phys. Lett. B 471 (1999) 308.

[11] ALEPH Collaboration, A. Heister, et al., CERN-EP/2002-026, Phys. Lett. B, submitted for publication, 2002.

[12] D0 Collaboration, V.M. Abazov, et al., Phys. Rev. Lett. 88 (2002) 171802.

[13] OPAL Collaboration, K. Ahmet, et al., Nucl. Instrum. Methods A 305 (1991) 275;

S. Anderson, et al., Nucl. Instrum. Methods A 403 (1998) 326; B.E. Anderson, et al., IEEE Trans. Nucl. Sci. 41 (1994) 845.

[14] E. Accomando, et al., in: G. Altarelli, T. Sjöstrand, F. Zwirner (Eds.), Physics at LEP2, in: CERN, Vol. 2, 1996, p. 299.

[15] T. Sjöstrand, Comput. Phys. Commun. 82 (1994) 74.

[16] B. Andersson, et al., Phys. Rep. 97 (1983) 31.

[17] OPAL Collaboration, G. Alexander, et al., Z. Phys. C 69 (1996) 543.

[18] C. Peterson, D. Schlatter, I. Schmitt, P.M. Zerwas, Phys. Rev. D 27 (1983) 105.

[19] S. Jadach, B.F. Ward, Z. Wạs, Phys. Lett. B 449 (1999) 97; S. Jadach, B.F. Ward, Z. Wass, Comput. Phys. Commun. 130 (2000) 260.

[20] S. Jadach, W. Płaczek, B.F.L. Ward, in: G. Altarelli, T. Sjöstrand, F. Zwirner (Eds.), Physics at LEP2, in: CERN, Vol. 2, 1996, p. 286.

[21] R. Engel, J. Ranft, Phys. Rev. D 54 (1996) 4244.

[22] G. Marchesini, et al., Comput. Phys. Commun. 67 (1992) 465.

[23] J.A.M. Vermaseren, Nucl. Phys. B 229 (1983) 347.

[24] J. Fujimoto, et al., Comput. Phys. Commun. 100 (1997) 128.

[25] S. Jadach, W. Płaczek, M. Skrzypek, B.F. Ward, Z. Wa̧s, Comput. Phys. Commun. 119 (1999) 272.

[26] J. Allison, et al., Nucl. Instrum. Methods A 317 (1992) 47.

[27] S. Catani, et al., Phys. Lett. B 269 (1991) 432.

[28] R.D. Cousins, V.L. Highland, Nucl. Instrum. Methods A 320 (1992) 331.

[29] Particle Data Group, D.E. Groom, et al., Eur. Phys. J. C 15 (2000) 838. 